\title{
Compression and Suppression of Shifting Receptive Field Activity in Frontal Eye Field Neurons
}

\author{
Wilsaan M. Joiner, ${ }^{1,2 *}$ James Cavanaugh, $^{1 *}$ and Robert H. Wurtz ${ }^{1}$ \\ ${ }^{1}$ Laboratory of Sensorimotor Research, National Eye Institute, National Institutes of Health, Bethesda, Maryland 20892-2510 and ${ }^{2}$ George Mason \\ University, Department of Bioengineering, Fairfax, Virginia 22030
}

Before each saccade, neurons in frontal eye field anticipate the impending eye movement by showing sensitivity to stimuli appearing where the neuron's receptive field will be at the end of the saccade, referred to as the future field (FF) of the neuron. We explored the time course of this anticipatory activity in monkeys by briefly flashing stimuli in the FF at different times before saccades. Different neurons showed substantial variation in FF time course, but two salient observations emerged. First, when we compared the time span of stimulus probes before the saccade to the time span of FF activity, we found a striking temporal compression of FF activity, similar to compression seen for perisaccadic stimuli in human psychophysics. Second, neurons with distinct FF activity also showed suppression at the time of the saccade. The increase in FF activity and the decrease with suppression were temporally independent, making the patterns of activity difficult to separate. We resolved this by constructing a simple model with values for the start, peak, and duration of FF activity and suppression for each neuron. The model revealed the different time courses of FF sensitivity and suppression, suggesting that information about the impending saccade triggering suppression reaches the frontal eye field through a different pathway, or a different mechanism, than that triggering FF activity. Recognition of the variations in the time course of anticipatory FF activity provides critical information on its function and its relation to human visual perception at the time of the saccade.

\section{Introduction}

We make saccadic eye movements up to three times per second, and with each saccade the visual image is displaced on the retina. Yet we maintain our perception of a stable visual world. One proposal is that anticipatory shifts of visual sensitivity accompany each saccade, and that these shifts are critical for visual stability (for review, see Wurtz, 2008). Visual sensitivity shifts from the current receptive field (RF) of a neuron to the location the RF will occupy after the saccade-the future field (FF) of the neuron. Neurons with these shifting RFs were first found by Duhamel et al., (1992) in monkey parietal cortex, and have subsequently been found in monkey frontal eye field (FEF; Umeno and Goldberg, 1997; Sommer and Wurtz, 2006). Recent psychophysical evidence has also suggested that these shifting RFs contribute to a perceived compression of the temporal and spatial locations of visual stimuli at the time of the saccade, and that this compression could also contribute to our perceived visual stability (Burr and Morrone, 2012; Cicchini et al., 2013).

The anticipatory shifts of sensitivity are not instantaneous but appear and disappear over time at the FF location. By determin-

Received July 11, 2013; revised Sept. 24, 2013; accepted 0ct. 1, 2013.

Author contributions: W.M.J., J.C., and R.H.W. designed research; W.M.J. performed research; W.M.J. and J.C. analyzed data; W.M.J., J.C., and R.H.W. wrote the paper.

Supported by the National Eye Institute Intramural Research Program at the National Institutes of Health. We are grateful to Altah Nichols and Tom Ruffner for machine shop support.

*W.M.J. and J.C. contributed equally to this work.

Correspondence should be addressed to Wilsaan M. Joiner, Department of Bioengineering, George Mason University, Nguyen Engineering Building, 1G5, 4400 University Drive, Room 3800, Fairfax, VA 22030. E-mail: wjoiner2@gmu.edu.

DOI:10.1523/JNEUROSCI.2964-13.2013

Copyright $\odot 2013$ the authors $\quad 0270-6474 / 13 / 3318259-11 \$ 15.00 / 0$ ing the rate of FF development, we should be able to better determine its role in perception and control of movement. Unfortunately, we have only minimal information on this time course. For FEF, we only know that different neurons have different FF latencies and waveforms (Sommer and Wurtz, 2008). For lateral intraparietal (LIP) area, we know the time course of FF activity averaged across a sample of neurons but not the individual variations (Kusunoki and Goldberg, 2003).

In our current experiments, we determined the time course of the FF activity for individual neurons in FEF. After identifying FEF neurons showing FF activity, we flashed stimuli lasting $50 \mathrm{~ms}$ in the FF of the neuron over a range of times right before the monkey made saccades. We found a remarkable variation in the latency and time course of FF activity among neurons. Also, FF activity was compressed in time compared with the spread of the stimulus flashes; the span of the flashes exceeded the span of the FF activity. We also found a striking saccadic suppression superimposed on the FF activity that has not been reported previously in FEF. To varying extents, the compression and suppression were overlapping in time. To determine the time courses of each, we constructed a model that was fitted to the data from each neuron. From this model we inferred the start, peak, and duration for both FF activity and suppression for each neuron. While both FF activity and suppression were synchronized to the saccade, their different time courses indicated the likelihood of separate mechanisms, or at least separate pathways.

\section{Materials and Methods}

Three adult male monkeys (Macaca mulatta) weighing from 7 to $9 \mathrm{~kg}$, were implanted with scleral search coils for measuring eye position, re- 
cording cylinders for accessing neurons in FEF, and a receptacle for receiving a post immobilizing the head during experiments as described previously (Sommer and Wurtz, 2000). All procedures were approved by the Institute Animal Care and Use Committee and complied with Public Health Service Policy on the humane care and use of laboratory animals. Recording procedures. We implanted a recording cylinder over the FEF (the anterior bank of the arcuate sulcus) approximately normal to the cranial surface. Cylinder placement over the sulcus was guided by magnetic resonance images. We recorded single neuron responses and microstimulated in FEF with tungsten microelectrodes advanced by a stepper microdrive. FEF neurons were verified using two criteria: saccade-related activity and the ability to evoke saccades with stimulation currents of $\leq 50 \mu \mathrm{A}$ (Bruce et al., 1985).

The monkey sat in a primate chair with its eyes $57 \mathrm{~cm}$ in front of a tangent screen. The chair was in the center of magnetic field coils in a dark room that was sound attenuated. Computers running REX (Hays, 1982) and associated programs controlled stimulus presentation, administration of reward, the recording of eye movements and single neuron activity, and the on-line display of results. We excluded neurons from analysis if we were unable to determine the parameters of the visual RF due to loss of neuronal isolation during the recording session.

Visual stimuli on the screen appeared on a gray background, backprojected by a DLP projector. In each experiment we first determined the center of the RF by creating a coarse spatial map. While the monkey fixated a central white cross, we sequentially presented spots of light of a fixed diameter ( 1 to $5^{\circ}$, depending on RF eccentricity) at nine locations on a $3 \times 3$ grid. The exact time of stimulus onset and offset was measured by a photocell on the projector side of the screen that registered the appearance of a white square in the corner of the screen (hidden from the monkey) for a single monitor cycle that was synchronized to both the appearance and disappearance of each stimulus. The stimulus grid center and spacing were adjusted to sample a large part of the RF and form an initial quantitative estimate of RF center and qualitative estimate of the extent. From the $3 \times 3$ grid, we determined the RF center as the average of the neuronal responses at nine locations weighted by the magnitude of the visual responses at each location.

We searched for neurons that had an identifiable visual RF, and then searched for that fraction that showed anticipatory visual activity as the monkey prepared to make a saccade. These neurons have shifting RFs: sensitivity to visual stimulation shifting from the spatial location of its current RF to the location that field will occupy after the saccade is completed. To determine whether a neuron possessed a shifting RF we systematically compared the neuronal responses to stimuli in its RF and the FF, that area that will be the RF after the saccade. We used a visually guided saccade task to study shifting RFs (see Fig. 1 $A, B$ ). On each trial, after $500 \mathrm{~ms}$ of initial fixation, a $50 \mathrm{~ms}$ visual stimulus was flashed in the center of the RF to determine the RF response. Following a variable delay $(500-700 \mathrm{~ms})$, the fixation point was turned off as a saccade target (the future fixation point) came on. Before the monkey made a saccade to the new fixation point, the same stimulus used to probe the RF was flashed in the FF. This $50 \mathrm{~ms}$ FF flash was at the same location in the FF as the flash had been in the RF. For example, if the center of the RF was at $x=-5^{\circ}, y=10^{\circ}$, and the monkey was required to make a saccade from the fixation point at $0^{\circ}, 0^{\circ}$ to a target at $15^{\circ}, 0^{\circ}$, the FF center would be at $10^{\circ}, 10^{\circ}$ (Fig. $1 A$ ). Importantly, the FF stimulus was extinguished before the saccade was made; trials with early saccades that started before or during the FF stimulus were discarded. The monkey received a liquid reward for maintaining fixation within a $1.5^{\circ}$ window around the fixation point at the start of the trial and for reaching the new fixation point within a $2.5^{\circ}$ window around the saccade target at the end of the trial.

Analysis of FF activity. We recorded from 192 neurons and concentrated on 67 of these that had shifting RFs. We did complete experiments on 28 of these neurons ( 16 in monkey Art, 10 in monkey Fln, 2 in monkey Cap). The remaining neurons were rejected because of the following: (1) response isolation became uncertain, (2) there was not sufficient variability in the probe and saccade timing for analysis (31 neurons), or (3) the RF was so large that the FF and RF overlapped, even with large saccades. In this latter case the FF flash activated the RF as well, as indi- cated by a fixed latency response after the FF stimulus onset, rather than a fixed latency after the saccade onset. We used results from all FEF neurons that had both a clear visual response and a shifting RF, whether or not there was accompanying saccade-related activity, as the presence (or absence) of purely saccade-related activity had no bearing on the presence of FF activity. Note that we use the term FF activity rather than FF response to emphasize the difference between this shifting RF activity with its dependence on the saccade, and a straightforward visual response.

To determine the dependence of FF activity on the timing of stimuli in the FF, we systematically varied the time of the FF flash before the saccade. We flashed the FF probes at a series of $50 \mathrm{~ms}$ intervals, from $50 \mathrm{~ms}$ to $\sim 250 \mathrm{~ms}$ before saccade onset. Therefore, the offset of the $50 \mathrm{~ms}$ duration probes varied from approximately saccade onset $(0 \mathrm{~ms})$ to 200 ms before the eye movement. Probe timing was synchronized to the offset of the fixation point, adjusted to the usual latency of the saccade. Because of variability in saccade latency, there was a continuum of probe times relative to saccade onset. Trials in which the saccade began during the FF probe were discarded because any eye movement would displace the location of the probe on the retina. Saccade initiation was identified as the time that eye velocity and acceleration exceeded 50 and $2000^{\circ} / \mathrm{s}^{2}$, respectively.

We divided the continuum of probe times into three successive time bins in each experiment, with each bin containing the same number of probe offset times. We used the probe offset time (which occurred $50 \mathrm{~ms}$ after the probe appeared) to provide a continuum of times contiguous with saccade onset. For each experiment, our goal was to obtain bins of probe times with both a sufficient number of trials and a reasonable spread of mean probe times. Over our 28 experiments, the mean number of trials in each of the three bins was 133, ranging from 14 to 389 trials in each bin. The purpose in binning the probes was to convert the continuum of probe times into a smaller number of cases each with an adequate number of trials such that effects of timing could be sufficiently measured. We used the mean probe offset time within each bin rather than the bin edges or centers, because the means introduce no systematic inconsistencies between the probe times and the expected timing of the FF activity. That is, given a range of probe times and a constant response latency, the mean responses to binned probes will naturally align (at some fixed offset) with the mean times in each bin rather than the bin edges or centers.

Because FF activity is synchronized to saccade onset (Sommer and Wurtz, 2008), we analyzed perisaccadic neuronal activity, beginning 200 $\mathrm{ms}$ before saccade onset and ending $300 \mathrm{~ms}$ after onset, lasting a total of $500 \mathrm{~ms}$. Neuronal activity was calculated using spike density histograms smoothed with a Gaussian with a $10 \mathrm{~ms}$ SD. We first determined the peak time of the FF activity within the specified $500 \mathrm{~ms}$ window around the saccade. We located the peak activity, and looked forward and backward in time to determine when the firing rate fell to $80 \%$ of this peak. The duration of the epoch when activity was at least $80 \%$ of maximum was taken to represent the width of the activity. We calculated the time of the activity peak by weighting the times within this epoch by the instantaneous firing rate at each time, in effect creating a weighted average of the center of the peak activity. For eight neurons with especially variable responses, we calculated the center and width using a $50 \%$ of peak activity epoch. This permitted us to calculate peak times for noisier and slow-rising activity that would otherwise produce spurious timing measures. We visually inspected each of the calculated peaks to confirm their validity.

We calculated the magnitude of each neuron's FF activity as the mean firing rate in a time window $100 \mathrm{~ms}$ wide centered on the weighted peak time. Using a fixed time window centered on the weighted peak provided a reasonable measure of the magnitude of activity that was not affected by its duration or decay. Visual responses to the RF probes were calculated in the same manner.

Significances and $p$ values, unless otherwise specified, were determined by $t$ tests. 
A

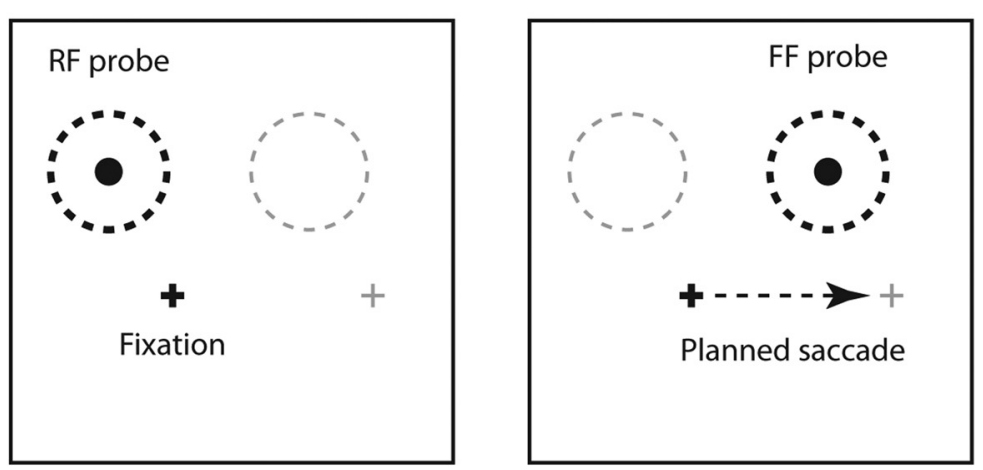

B

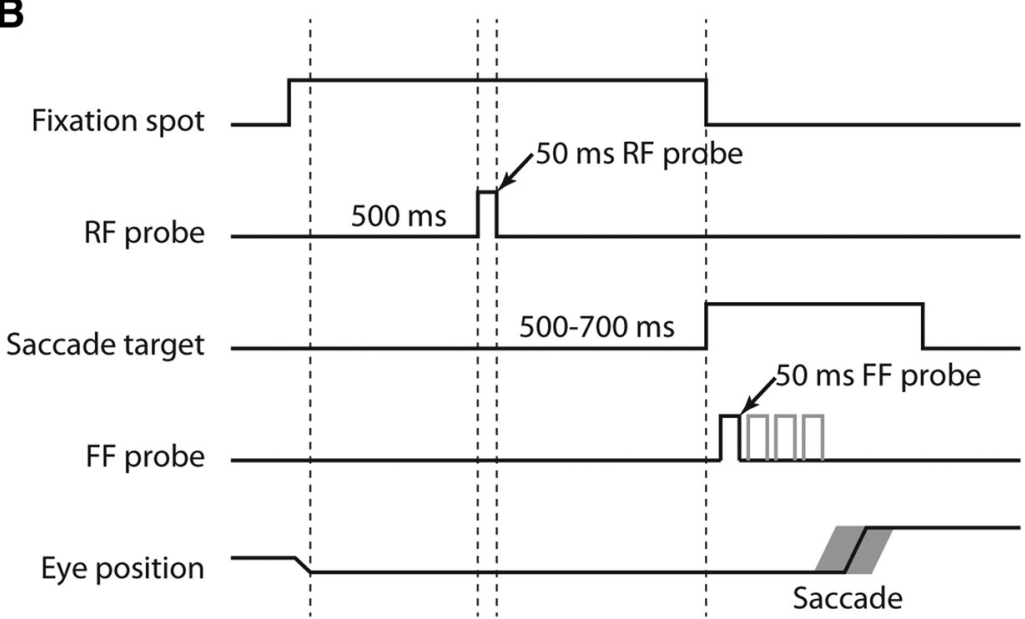

Figure 1. Behavioral paradigm for studying the temporal characteristics of shifting RFs. A, Position of stimuli for determining RF and FF activity. During fixation (eye position is shown by the small black cross) a probe stimulus was flashed in the center of the RF (left, black dashed region and the probe therein). The fixation point was extinguished as a peripheral target came on (represented by the gray cross), signaling to make a saccade. Just before the saccade (right, dashed arrow) the same probe stimulus was flashed in the center of the FF of the neuron (right, black dashed circle). B, Timing of RF and FF stimuli. On every trial, after $500 \mathrm{~ms}$ of fixation, there was a probe flashed for $50 \mathrm{~ms}$ in the RF. After an additional $500-700 \mathrm{~ms}$ the fixation point went off and a peripheral target appeared, signaling the monkey to make a saccade to the target. After the target appeared, but before the saccade, a $50 \mathrm{~ms}$ probe was flashed in the FF at one of up to four times after the target appeared. These times are represented by the four deflections on the FF Probe time line. Possible probe times were separated by $\sim 40-50 \mathrm{~ms}$. There was variability in the saccade timing (depicted by the gray outline on the Eye Position line), which contributed to the range of probe-to-saccade times. Only one stimulus in the RF and FF was flashed on each trial.

\section{Results}

\section{Confirmation of FF activity}

To be sure that each of the 28 neurons we analyzed displayed FF activity, we identified the two primary salient characteristics of FF activity: it depends on both preparations to make a saccade and a stimulus in the FF. To evoke FF activity from each FEF neuron, we visually stimulated twice as the monkey fixated before the saccade (Fig. 1). The first stimulus was a $50 \mathrm{~ms}$ spot that came on in the RF. For the example neuron in Figure 2 the visual response (data not shown) had a mean latency of $61 \mathrm{~ms}$ from the stimulus onset. As the monkey prepared to make a saccade to the target, a second $50 \mathrm{~ms}$ stimulus flashed in the FF. This second probe also resulted in neuronal activity as indicated by the black trace for the example neuron in Figure $2 A$. However, the FF probe elicited no activity on trials when the monkey simply fixated and no saccade was being prepared (Fig. $2 A$, dashed trace), indicating that the FF activity depended on saccade initiation. The FF activity from Fig. $2 A$ is shown again in Figure $2 B$, now synchronized to the saccade (Fig. $2 B$, thick trace). When there was no stimulus before the saccade, there was no activity in the FF (Fig. $2 B$, thin trace). To be classified as having FF activity, we required FEF neurons to exhibit both these characteristics.

For the 28 neurons with FF activity that fit these criteria, we confirmed the importance of the saccade by expanding upon the finding that FF activity is better aligned to saccade onset than to stimulus onset (Kusunoki and Goldberg, 2003; Sommer and Wurtz, 2008). Although this has often been shown for single neurons, we adopted a more quantitative approach. For each neuron, we examined the magnitude and the width of FF activity aligned to both the saccade and the probe (see Materials and Methods for determination of activity peak and width). To compare magnitude, we used a contrast measure using both the magnitude of the FF activity aligned to the saccade $\left(M_{S}\right)$ and the probe $\left(M_{P}\right)$. We calculated this relative activity $M_{S P}$ as $\left(M_{S}-M_{P}\right) /\left(M_{S}+M_{P}\right)$. We similarly used width of the saccadealigned activity $\left(W_{S}\right)$ and the probealigned activity $\left(W_{P}\right)$ to calculate the relative saccade-aligned activity width $\left(W_{S P}\right)$ as $\left(W_{S}-W_{P}\right) /\left(W_{S}+W_{P}\right)$.

The abscissa of Figure $2 C$ shows $M_{S P}$ : the relative magnitude of the FF activity aligned to the saccade. The distribution at the top of Figure $2 C$ shows how responses tended to be greater when aligned to the saccade. The ordinate of Figure $2 C$ shows $W_{S P}$ : the relative width of the FF activity aligned to the saccade. The histogram on the right of Figure $2 C$ shows how saccadealigned activity tended to be narrower than when aligned to the probe. Overall the preferred alignment of FF activity to the saccade is evident in the greater magnitude $(+5.2 \% \pm 1.5 \mathrm{SEM}, p=0.002)$ and narrower width $(-11.2 \% \pm 3.5 \mathrm{SEM}$, $p=0.005)$ when aligned to the saccade, quantifying this observation.

As a final point, the magnitude of the FF activity was on average only $56 \%$ ( \pm 5.5 SEM) of the magnitude of the RF response. Interestingly, the magnitude of the FF activity was not correlated with the magnitude of the RF response $(r=0.250, p=0.20)$.

The above results are clear confirmation that the FF activity we study is a saccade-related phenomenon. FF activity does not exist without a saccade, and the saccade alone (in the absence of a probe) does not evoke FF activity. Finally, FF activity aligns better to the saccade than the stimulus that evokes it.

\section{Temporal dependence of the magnitude of FF activity}

A major goal of our experiments was to determine the effect of the time of FF stimulation on the magnitude and the timing of the resulting FF activity. To do this we varied the interval between a brief stimulus probe $(50 \mathrm{~ms})$ and saccade onset, and then determined the variation in the timing of the FF activity. We flashed the FF stimulus at up to four different times before the saccade (see Materials and Methods; Fig. 1B). Additional variation in the monkey's saccade latency produced a continuous distribution of 

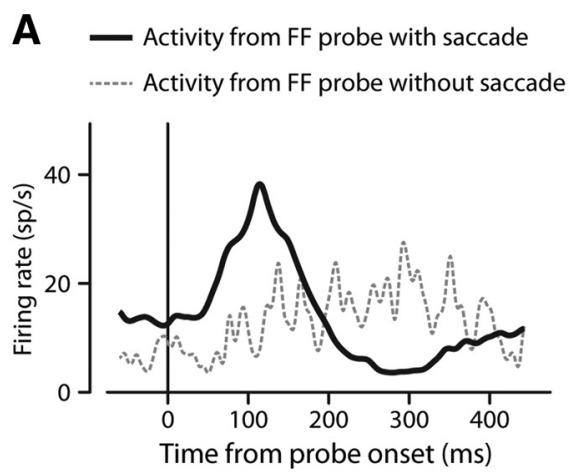

B
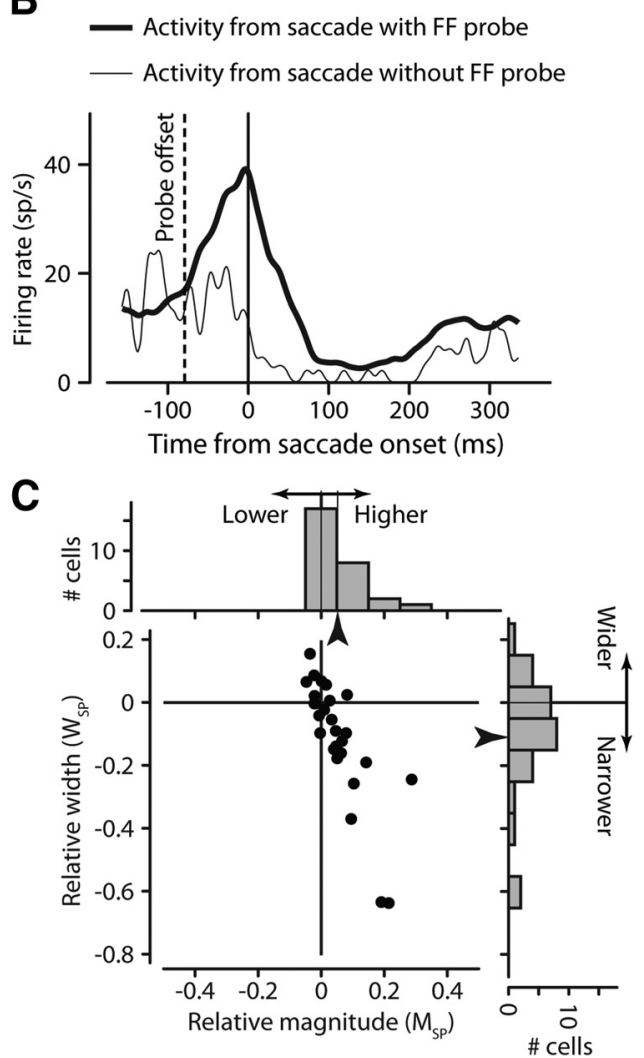

Figure 2. Confirmation of two primary characteristics of $\mathrm{FF}$ activity. FF activity depends on both preparation to make a saccade $(\boldsymbol{A})$ and a stimulus in the $\mathrm{FF}(\boldsymbol{B})$. For an example neuron, $\mathrm{FF}$ activity is aligned on the FF probe at time $0(\boldsymbol{A})$, and shows activity when there is a saccade (solid black trace) and little activity without the saccade (dashed gray trace). The same FF activity, now aligned on saccade onset (B), shows FF activity (thick black trace) when there is an FF stimulus, but not when there is no stimulus (thin trace). C, FF activity is better aligned to saccade onset than to stimulus onset. For each neuron, we calculated the change in FF activity magnitude (abscissa) and FF activity width (ordinate) as a contrast measure representing how each value changed when aligned to the saccade. Points tend to fall below (narrower) and to the right (higher) of zero, indicating significantly larger and narrower patterns of activity when aligning FF activity to the saccade rather than the probe time. Marginal histograms show the distributions of these values.

probe times. For each neuron, we divided this time continuum into three successive bins, with each bin containing the same number of probe offset times. The colored regions on the left side of Figure 3, $A$ and $B$, demonstrate this binning for two example neurons. Each color corresponds to one of the three bins, and the width of each color is the range of the probes within that bin. Note that the red colored bin extends all the way to saccade onset, as we are binning the probe offset times. The three vertical dashed lines are color coded to each bin and show the mean probe offset time for that bin.

In Figure 3, $A$ and $B$, the spike density histograms of neuronal activity resulting from probes in each of the three bins are color coded to that bin. The peak activity times for each bin are indicated by the colored spots above the spike density functions. The left side of Figure $3 A$ shows how the saccade-aligned activity evoked by probes in different bins varies both in magnitude and timing. The probes for the neuron shown in Figure $3 \mathrm{~A}$ produce FF activity before and during the saccade whereas the probes in Figure $3 B$ produce FF activity after the saccade. The right side shows the same FF activity as on the left, but aligned to the probe offset times. These two examples demonstrate the variability in FF activity that we exploit to study the relationship between FF activity and both of its essential components: the probe and the saccade.

\section{Temporal compression of FF activity}

If the activity in the FF was a simple visual response, we would expect FF activity to occur at a fixed latency after the probe. Such probe-determined activity, when viewed relative to the saccade, would span the same time period as the probes. We can therefore explore the relative dependence of FF activity timing on both the probe and on the saccade by examining the spread of the activity when aligned to the saccade or the probe.

Consider again the FF activity shown on the left side of Figure $3 A$ where activity is aligned to the saccade. Although the mean times of the FF probes (shown by the dashed colored lines) occurred over a $77 \mathrm{~ms}$ range before the saccade, the range of the peak FF activity (marked by the colored spots above the spike density functions) spanned $30 \mathrm{~ms}$, less than half the span of the probes. Interestingly, when the FF activity is aligned to the probe offset times (Fig. $3 A$, right), the resulting spike density histograms span $51 \mathrm{~ms}$ (again indicated by the three colored dots above the spike density functions on the right side of Fig. $3 A$ ).

The neuron shown in Figure $3 A$ is representative of neurons that had FF activity before and during saccade onset. But it has previously been shown that different FEF neurons have different FF activity latencies (Sommer and Wurtz, 2008). Compare Figure $3 A$ with the neuron in Figure $3 B$, which shows FF activity after the saccade. For this example, the mean probe times spanned $72 \mathrm{~ms}$, but the resulting FF activity spanned only $3 \mathrm{~ms}$ when aligned to the saccade (left). Although the saccade-aligned activity in Figure $3 B$ was compressed in time, the spread of FF activity when aligned to the probe was nearly as long as the spread of probe times (70 $\mathrm{ms}$, right). In both example neurons in Figure 3, the spread of FF activity was compressed in time compared with the span of the FF probe stimuli.

Figure $3 C$ shows this compression for the 28 neurons in our sample by comparing the time span of stimulus presentation to the time span of the FF activity. The abscissa shows the span of the mean probe times aligned on saccade onset (the span of the vertical dashed lines on the left side of Fig. $3 A, B)$. The ordinate of Figure $3 C$ shows the span of the FF activity peaks when aligned to the saccade onset (the span of the colored dots on the left). The example neurons presented in Figure $3, A$ and $B$, are distinguished by black outlines in Figure $3 C$. If the span of the FF activity peaks were as wide as the span of the probes before saccade onset (as would be expected without compression), the points would fall on the diagonal line indicating equal spans. However, as in the example neurons, nearly all of the points fall below the line. Across the sample, the mean span of the peak FF activity was $17 \pm 3 \mathrm{~ms}(\mathrm{SE})$ and the mean span of the probes 
A
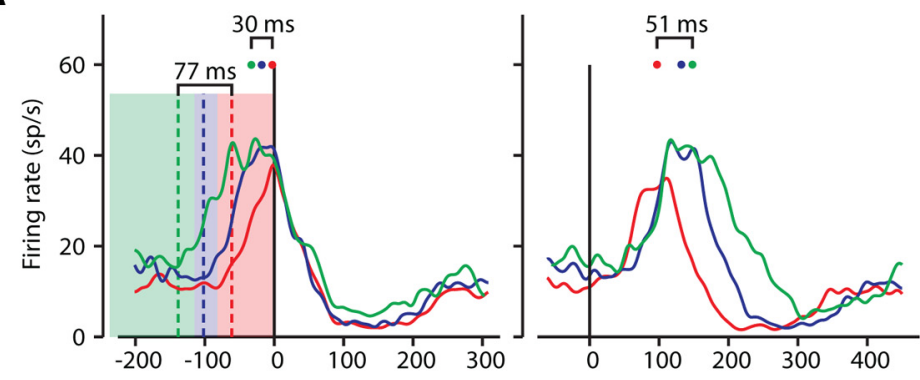

B
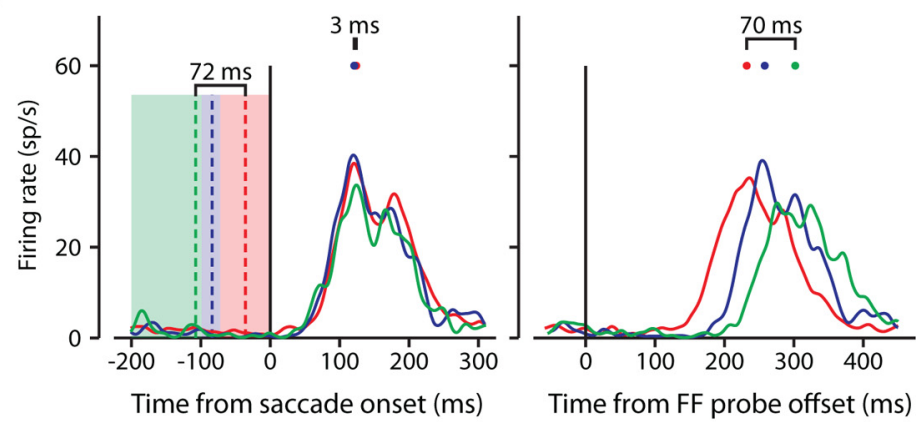

C

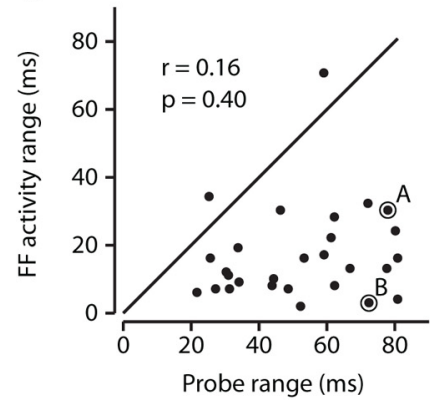

D

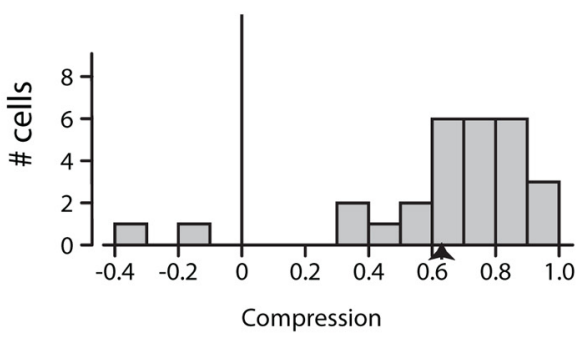

Figure 3. Examples of temporal compression of FF activity. Two example neurons with FF activity occurring during $(\boldsymbol{A})$ and after $(B)$ the time of the saccade. In the left column of $\boldsymbol{A}$ and $\boldsymbol{B}$, the three colored regions represent the ranges of probe offset times in each of three bins with equal numbers of trials. For the example neuron in Figure $2 A$ the $237 \mathrm{~ms}$ range was divided into three bins of 332 trials each. The red region represents the range for probes ending closest to saccade onset, between 82 and $0 \mathrm{~ms}$ before the saccade, the blue between 115 and $82 \mathrm{~ms}$, and the green between 237 and $115 \mathrm{~ms}$ before the saccade. The colored dashed line within each bin is the mean probe time within that bin, and the range from the earliest to the latest mean probe times is indicated above the lines. The solid colored traces show the saccade-aligned FF activity associated with the FF probes in bins of the same color. For example, the red trace resulted from the probes in the red bin. The color-coded points above each trace show the peak of each, and the range of these peaks is above the traces. The range of activity peaks is compressed compared with range of the stimulus bins. The right columns of $A$ and $B$ show the same activity but now aligned to the probe offset. These peaks and ranges are also shown. C, Relationship between timing of probes and timing of $\mathrm{FF}$ activity for the sample of neurons. For 28 neurons, the range of the saccade-aligned FF activity peaks is plotted against the range of the mean probe times. Almost all points fall below the diagonal indicating that the range of $\mathrm{FF}$ activity was shorter than the range of the probes. Example cells from $\boldsymbol{A}$ and $\boldsymbol{B}$ are indicated. $D$, Distribution of temporal compression across our sample. Compression is shown as the reduction in the range of FF activity peaks relative to the range of probes [(probe range - activity range)/probe range].

before saccade onset was $52 \pm 4 \mathrm{~ms}$. For the sample of neurons, the span of the FF activity peaks was significantly less than the span of the probes before saccade onset (two-tailed $t$ test, $p \ll$ 0.001 ). This indicates a striking temporal compression of the FF activity across the sample of neurons.

We further quantified this reduction in the temporal spans by determining the temporal compression ratio: the proportional decrease of time span between the probes and the FF activity peaks (Fig. 3D). Here, a ratio near 0 represents no temporal compression, and a ratio close to 1 signifies almost total temporal compression when the peaks of the FF activities would span only a few milliseconds. For Figure $3 A$ compression would be (77$30) / 77=0.61$ and for Figure $3 B$ equaled $(72-3) / 72=0.96$. As shown in the figure, all but four neurons had a temporal compression $>40 \%$, with a mean temporal compression of $64 \%$ $(p \ll 0.001)$. In contrast, the probealigned FF activity (as on the right side of Fig. $3 A, B)$ did not demonstrate such pronounced temporal compression $($ mean $=$ $11 \%, p=0.27)$.

Our last question regarding temporal compression of FF activity was whether there was a relationship between the span of FF activity and the time of the saccade; did the peaks spread out more or less when the FF activity was closer to the saccade? We performed a correlation between the time of the peak FF activity (over all probes, aligned to saccade onset as in Fig. $2 B$ ), and the range of the peaks when the probe times were binned (as in Fig. 3) and found no significant relationship between the two $(r=0.25, p=0.21)$ suggesting that the compression of FF activity is not dependent on whether the activity occurred before, during, or after the saccade. These results summarize the prevalence of temporal compression of FF activity in FEF, compression that is equally strong whether it occurs during or after the saccade.

\section{Saccadic suppression of FF activity}

While examining FF activity in frontal eye field, we often observed a distinctive suppression of neuronal activity that has not been previously reported. Figure $4 A$ illustrates this suppressive influence for a sample neuron with striking suppression before the FF activity, while Figure $4 B$ shows a neuron with little visible suppression. Suppression is also evident for the neurons illustrated in Figure 3 (left): unmistakable in Figure $3 A$ (after the FF activity), and to a lesser extent in Figure $3 B$ (the saccade-aligned dips during the activity). As suggested by our examples, the variability of suppression over our sample was substantial. In the 28 neurons, 12 showed suppression as clear as that in Figure $4 A$, while 6 showed a lesser amount of suppression as in Figure 3B. For 10 neurons, suppression was virtually imperceptible (Fig. $4 B$ ).

The increase of neuronal activity at the time of the saccade in neurons with FF activity provided a higher background against which the suppression could often be seen. But it also led to an intermixing of FF activity and suppression that made it difficult to discern one change in neuronal activity from the other. Consequently, the patterns of activity we observed defied classification into specific categories (e.g., activity before/after saccade, strong/weak suppression, suppression before/during activity). We therefore developed a simple model that incorporated the distinctive aspects of FF activity and suppression that we observed. Our hopes were that the model would be able to separate in time the strengths and time courses of the FF activity and the 
accompanying suppression, and succeed in differentiating these two influences where simple visual inspection failed.

\section{Model of FF activity and suppression}

We developed a model comprising simple components based on our observations. We assumed that the probes create an underlying pattern of activity with a fixed latency from each probe. These patterns of activity were transiently revealed by the appearance and disappearance of the FF near the time of the saccade. Finally, the revealed probe activity could be affected by a suppressive influence aligned to the saccade.

The formalization of the model was simple. First we assumed that the FF probes created a slowly decaying pattern of activation, because we often recorded FF activity 300-400 ms after the $50 \mathrm{~ms}$ probes disappeared. We modeled the probe activity as a function that peaks relatively quickly and then decays; we chose an $\alpha$ function. Each neuron had a single $\alpha$ function, so the three bins of probes produced three identical $\alpha$ functions separated in time. This $\alpha$ function was specified by:

$$
\alpha_{p}(t)=\frac{\left(t-t_{0}\right)}{\tau} \exp \left(\frac{-\left(t-t_{0}\right)}{\tau}\right),
$$

where $\alpha_{p}$ is the $\alpha$ function for probe bin $p$, $\tau$ is the time constant, and $t$ is time. For each neuron, the $\alpha$ function incorporated a second parameter $t_{0}$ representing the latency of the induced activity. The separation of the three $\alpha$ functions in time was set by the mean probe times in each bin, and was fixed for each cell. We normalized the $\alpha$ functions to a height of 1 .

Second, we modeled the transitory presence of the FF as a time-varying envelope of sensitivity to the probe activity with a Gaussian temporal profile:

$$
f f(t)=\exp \left(\frac{-\left(t-\mu_{f f}\right)^{2}}{2 \sigma_{f f}^{2}}\right),
$$

in which $\mu_{f f}$ is the time at which the sensitivity peaks, and the standard deviation $\sigma_{f f}$ determines the duration of the FF.

Third, the suppressive influence was similarly modeled with a Gaussian temporal profile of a decrease in activity:

$$
s(t)=1-k_{s} \exp \left(\frac{-\left(t-\mu_{s}\right)^{2}}{2 \sigma_{s}^{2}}\right),
$$

where we have added the scale term $k_{s}$ permitting various strengths of suppression.

The complete model of the activity evoked by probe $p$ is now the following:

$$
r_{p}(t)=s(t) \cdot\left(r_{0}+k_{r} \cdot f f(t) \cdot \alpha_{p}(t)\right) .
$$

We multiply the probe $\alpha$ function by the FF Gaussian. We then scale this curve (multiply by $k_{r}$ ) and add a baseline firing rate $\left(r_{0}\right)$. Finally we multiply by the suppressive Gaussian.
Figure 5 shows the results of fitting this model to the four example neurons shown so far. The three curves for each neuron representing FF activity were fit simultaneously so a single set of parameters generated all three fit curves for the three probe times. In other words, the only difference in the three different colored curves is the relative timing of the probes, which was extracted from the data and fixed during the fitting procedure for each neuron.

Figure $5 \mathrm{~A}$ (left) shows how the model fit the responses to our first example neuron in which the FF activity occurred before and during the saccade, and in which suppression affected the end of the FF activity. The model accounts for not only the different response timing for the different probes, but also for the different response magnitudes. The suppression also is represented in the fits.

For each neuron, the $R^{2}$ value is shown for the fit to the model. The right side of Figure $5 A$ graphically depicts the components of the model that fit these responses. The probe times, which were fixed, are denoted by the dashed vertical lines (Figs. 3, 4), and are color coded to their corresponding curves. The $\alpha$ functions representing the activity induced by the probes are shown as the solid colored lines. The solid black curve denotes the temporal envelope of the FF, and the black dashed curve shows the location in time and relative magnitude of the suppressive influence. In the model, the peak magnitude of the probe responses was scaled to 1 , as was the peak of the FF envelope. The height of the dashed curve represents the strength of the suppressive influence relative to the solid black FF envelope.

Figure $5 \mathrm{~A}$ shows the model accounting for a neuron with FF activity of different magnitudes and latencies. The three other examples (Fig. $5 B-D$ ) show the model's ability to capture various other patterns of FF activity. Figure $5 B$ shows the model representing FF activity after the saccade with a small amount of suppression during the activity. Figure $5 C$ shows strong suppression before the FF activity. Figure $5 D$ shows the fit to a neuron with no 
A

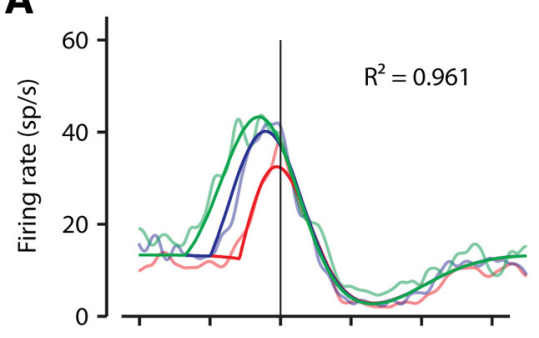

\section{B}
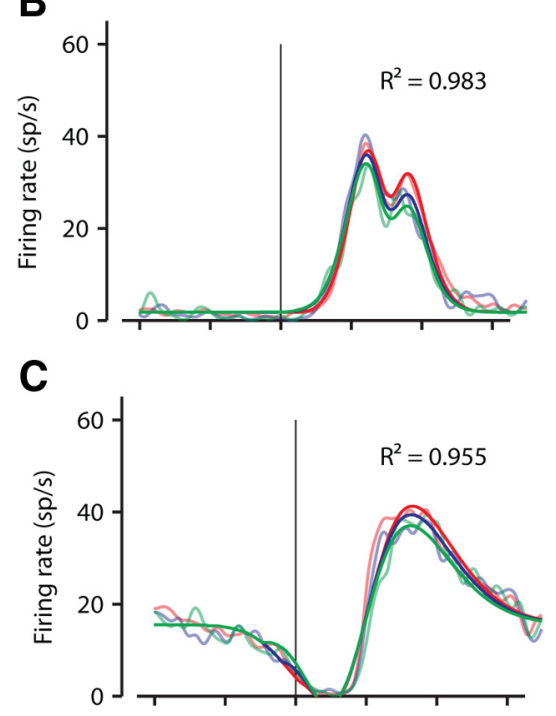

D

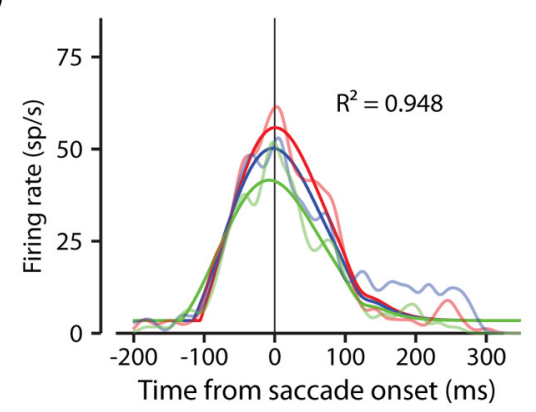

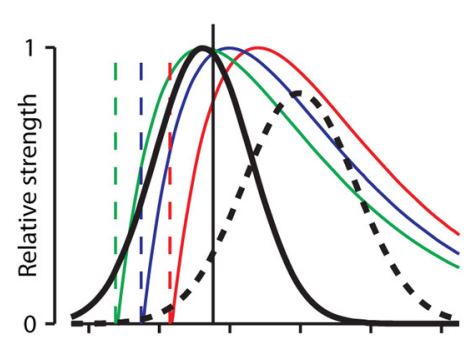
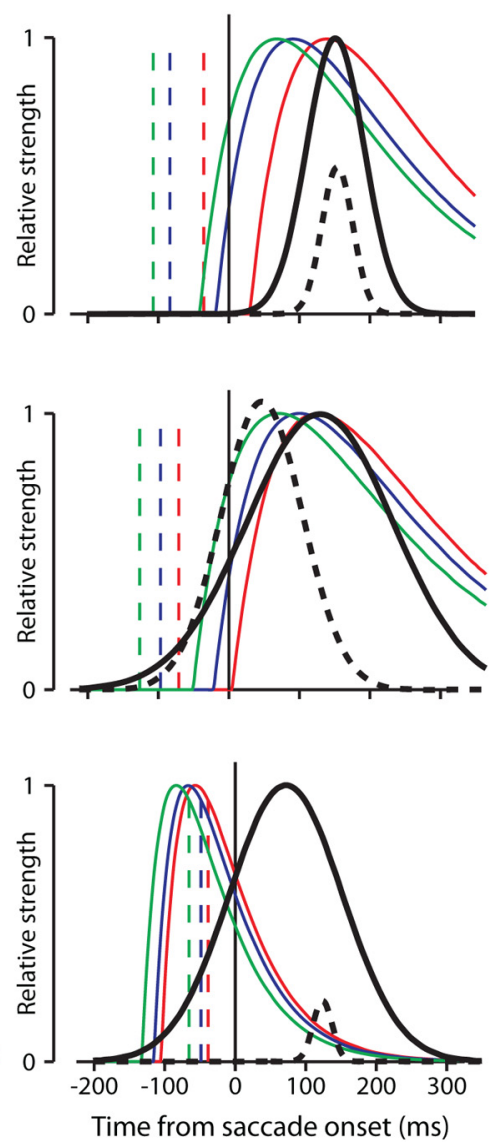

Figure 5. Fits of the model to sample neuron activity. $A-D$, Show the same four neurons as in Figures $3 A$ and $B$ and $4 A$ and $B$, respectively. Colors code for probe bins and curves are as in Figures 3 and 4 . Saccade-aligned responses are shown on the left. Superimposed on the FF activity are color-coded fits of the model (the bolder colored curves). Each example shows how well the model was able to account for a variety of aspects of the data. The $R^{2}$ values in each part indicate good fits. The right side graphically depicts the three parameters of the model: the slow decaying activity of the FF probe stimuli in three bins represented by $\alpha$ functions (colored curves), the Gaussian temporal profile of FF activity (solid black curve), and the Gaussian temporal profile of suppression (dashed black curve). The dashed vertical colored lines show the mean binned probe times for each neuron. The height of the dashed black suppression curve relative to the solid black FF curve represents the strength of the suppressive influence relative to the excitatory FF.

visible suppression. Keep in mind that for each neuron the only difference among the three curves fit to the data was the time of the probe; the same equation generated each curve.

Overall the model did well accounting for the variability in the characteristics we observed in FF activity, including differences in activity onset, activity magnitude, and the presence of saccaderelated suppression. The overall quality of the fits is quantified in Figure $6 A$, which shows the distribution of $R^{2}$ values across our sample. The shaded bars represent $R^{2}$ of at least 0.8 , indicating the fits that provided the most confident predictions of FF activity $(n=21)$. The model was also able to represent temporal compression of FF activity. Figure $6 B$ plots the temporal compression predicted by the model against that measured from the data. The points from the less confident fits $\left(R^{2}<0.8\right)$ are plotted as open symbols. The correlation between the data and the model was significant $(r=0.63, p<0.001)$. The marginal histograms show the distributions of compression from the data (top, duplicated from Fig. 3D) and the fits (right). Note that the model estimated higher compression than that shown by the data. We believe the higher estimation of compression from the model is more accurate due to the lack of activity variations (noise) that occur in the data. If compression is as high as the fits suggest, the variations in the data naturally would spread out the activity, making measurements of spread from the data larger, and estimates of compression correspondingly smaller.

It occurred to us that in some cases, such as in Figure 5D, suppression might not be necessary to account for some patterns of activity. To test this possibility we fit the data from each neuron to a version of the model without suppression. We simply left out the final step of multiplying by the suppressive influence (we omitted $s(t)$ in Eq. 4). We calculated an adjusted $\chi^{2}$ statistic (Hoel et al., 1971; Cavanaugh et al., 2002) for the fits with and without suppression to determine whether the improvement in fit quality warranted the addition of the extra parameters that represented the suppression. For all but two neurons (both with $\left.R^{2}<0.6\right)$, the adjusted $\chi^{2}$ errors for the model without suppression were higher than when including suppression; the addition of suppression improved the fit (even taking into account the additional parameters). Figure $6 \mathrm{C}$ shows the ratio between the $\chi^{2}$ errors from both fits for each neuron (no suppression/suppression). The marginal histogram at the top shows the distribution of these ratios. Larger values indicate the importance of suppression as a factor in fitting the data. Values less than one would indicate that suppression was not required to model FF activity. The ordinate shows the strength of suppression determined by the model. There was, as would be expected, a significant correlation between the strength of suppression and its importance in fitting the data $(r=0.51$, $p=0.006)$.

The variety of FF activities we observed was accounted for by a simple model in which the FF appears briefly near the time of the saccade. The probes create a predictable pattern of activity that is revealed when the FF appears. The addition of saccade-related suppression permitted the model to account for most of the variations in FF activity. If we regard our model as descriptive, we can consider the temporal envelope of increased sensitivity in the model to correspond to the appearance of the FF (as we in- 


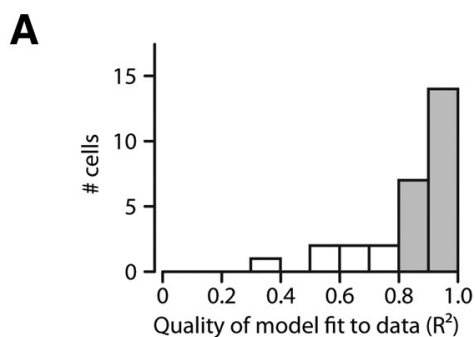

B

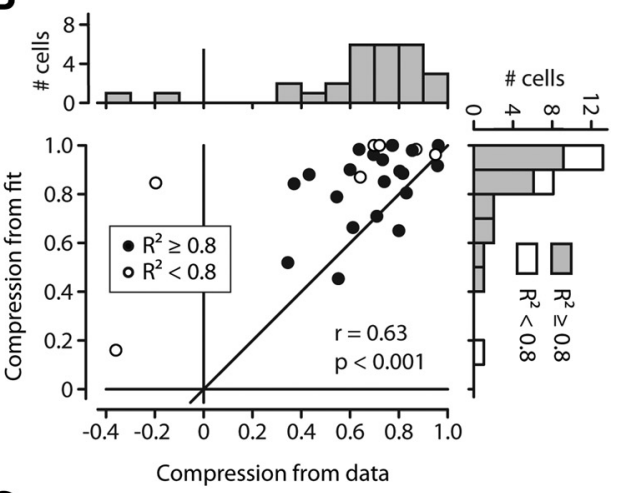

C

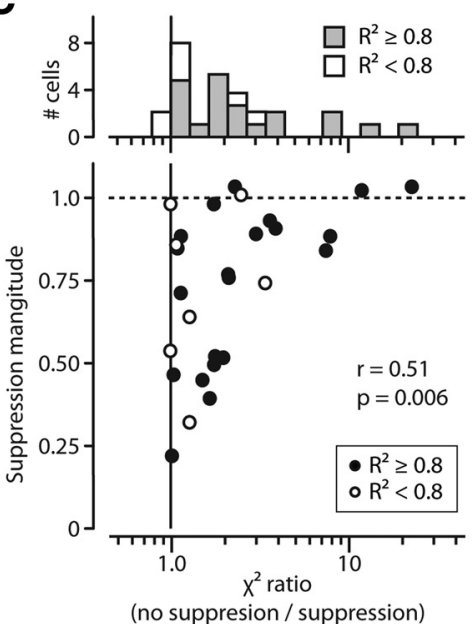

Figure 6. Evaluating the model fits for the sample of neurons. $A$, Distribution of $R^{2}$ values over all 28 neurons. Over our sample, 21 neurons had fits with an $R^{2}$ of 0.8 or better. $\boldsymbol{B}$, The model accounts for FF activity compression. For all 28 neurons, $\boldsymbol{B}$ shows the temporal compression of probes determined by the model versus the temporal compression determined from the data. The more confident fits $\left(R^{2} \geq 0.8\right)$ are plotted as solid black symbols, open symbols otherwise. C, Magnitude of suppression versus importance of suppression in model fits. For each neuron, the abscissa shows the ratio between the adjusted $\chi^{2}$ errors of models with and without suppression. The distribution of ratios is shown in the marginal histogram at top. Greater values show increased importance of suppression. The ordinate shows the strength of suppression as determined by the model ( $k_{s}$ in Eq. 3). Suppression was a critical component in FEF activity.

tended), and we can use the model to infer certain aspects of the mechanisms underlying FF activity.

\section{Model estimates of FF and suppressive influences}

By examining the components of our model as applied across the sample of neurons, we derived an estimate of the two key underlying features we have been exploring: the time course of FF sensitivity and that of the saccadic suppression. The time course of FF sensitivity is given by the center and width of the FF Gaussian in the model. Similarly, the time course of saccadic suppression is taken from the center and width of the model's suppressive Gaussian. Figure 7 shows these estimates.

Figure $7 A$ shows the distribution of times that FF sensitivity peaked. As in Figure 6, quantities from fits with $R^{2} \geq 0.8$ are shaded gray, and are white otherwise. The mean peak was $129 \mathrm{~ms}$ after the saccade, ranging from shortly before the saccade to well after the saccade. The duration (width at half-height) of the FF sensitivity according to the model (Fig. $7 B$ ) was on average 173 $\mathrm{ms}$ (note that the width at half-height of the Gaussian components corresponds to $\sim 2.35 \mathrm{SD}$ ). Figure $7, D$ and $E$, show the center and width of the suppression, the means of the peaks and the widths being 93 and $123 \mathrm{~ms}$, respectively.

To make informed speculations as to the origins of the FF activity we would want to know not just when the FF sensitivity peaks, but perhaps more importantly when it begins. Figure $7 C$ shows the start of the FF sensitivity (when it reached half of its maximum sensitivity). Naturally, the distribution of starts is shifted to earlier times relative to the peaks, making it clear that the FF often appears well before the saccade starts (Sommer and Wurtz, 2008; Fig. 4). Figure $7 F$ shows the beginning of the suppressive influence, which although distributed differently (note the peak) spans a similar range relative to the saccade as the FF sensitivity (Fig. 7C).

Although the time of the FF activity and the time of the suppression share similar distributions (Fig. $7 A, D$ ) there was little similarity on a cell-by-cell basis. Figure $7 G$ demonstrates this by showing the time when the FF sensitivity peaked versus the time when the suppression peaked, demonstrating the lack of correlation. In Figure $7 \mathrm{H}$, we examined the start of the sensitivity and the start of the suppression (rather than their peaks). There was a slightly stronger relationship $(r=0.42, p=0.025)$ although the correlation remained less than compelling.

Part of the reason for the decoupling of the time course of the FF activity and the suppression is demonstrated in Figure 8. Recall from Figure 2 that FF activity requires both (1) a saccade and (2) a probe in the FF. In the absence of a probe, there is no FF activity, but some neurons still exhibited varying amounts of purely saccade-related activity during other experiments (Fig. $8 A$, solid trace; same neuron as in Fig. 2). We often observed similar suppression during saccadic activity as we did during FF activity (Figs. 3, 4). Suppression during purely saccadic responses often followed the same time course as the suppression seen during FF activity. This is demonstrated by the dashed line in Figure $8 A$, which is the estimate of the saccadic suppression determined by the model for this cell. The suppressive component determined by the model's fit to the FF activity aligns with the suppression seen in the saccade-only activity. We explored a subset of our neurons with saccade-only activity for the presence of suppression during the saccade. By using criteria extracted from the model's fit and its parameters, we chose neurons with an $R^{2}$ of at least 0.8 , a saccade strength of at least 0.4 , and a $\chi^{2}$ ratio of at least 0.25 . This selected neurons for which FF activity was reasonably well fit with substantial and necessary suppression. Of the 11 neurons fitting these criteria, there were 8 for which we had sufficient data showing activity during the saccade only. We aligned the saccade-only activity to the center of the suppression relative to saccade onset as determined by the model's fit to the data. We normalized each neuron's activity and averaged. We also averaged the suppression as determined by the model. Using these averages of activity and suppression, Figure $8 B$ shows unmistakable suppression of activity when the model predicted it should occur. This suggests that the suppression is a saccadic phenomenon rather than a characteristic of the FF. But because FF activity 

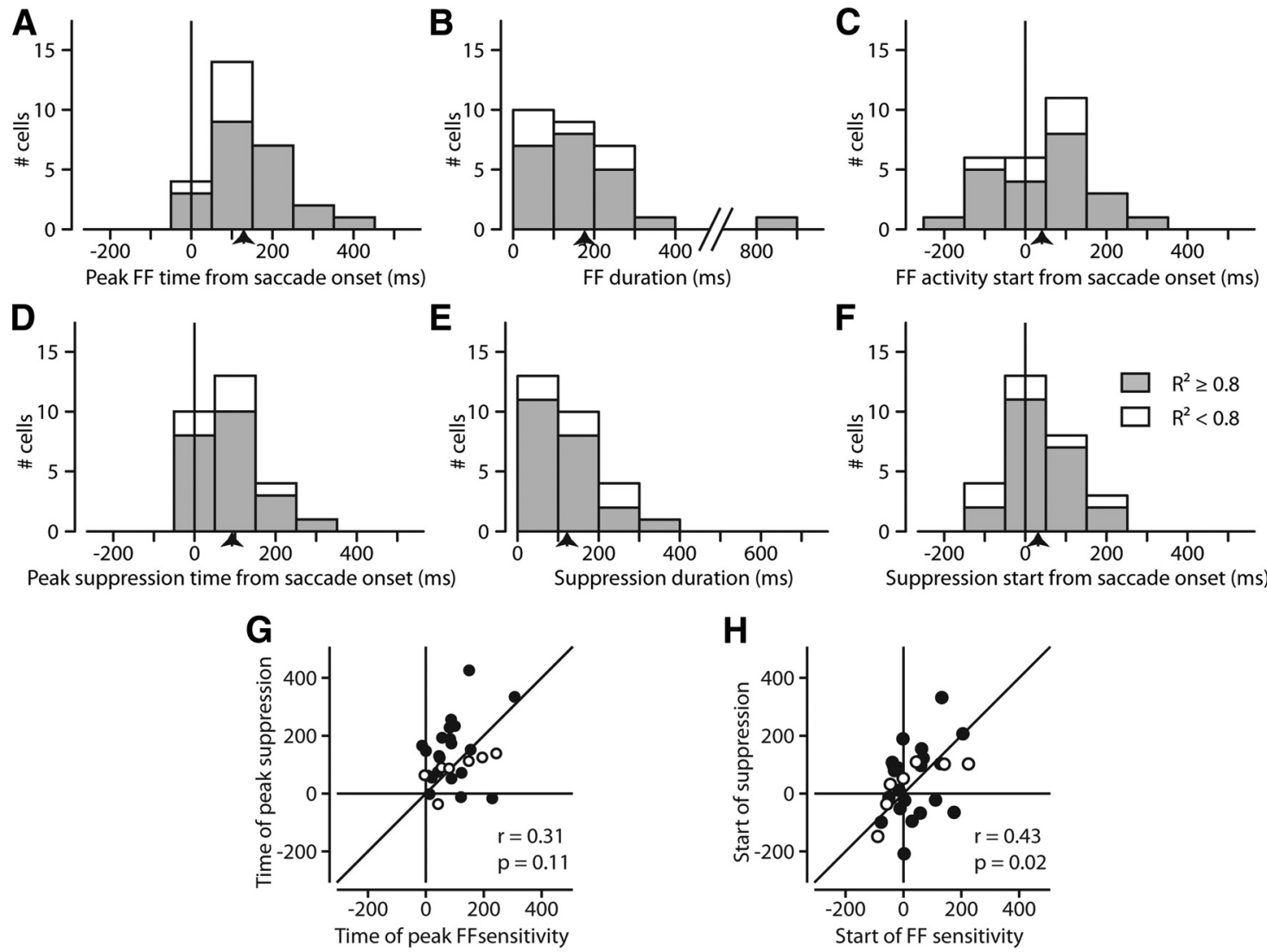

Figure 7. Model parameters reveal the timing of $F F$ and suppressive influences. $A$, Distribution of FF peaks. $A$, Shows, for our sample, when the model determined the times of peak $F F$ sensitivity. This was simply the center of the Gaussian representing the FF sensitivity. $\boldsymbol{B}$, Duration of FF sensitivity, how long the model determined the FF lasted. The duration is considered as the width at half-height of the FF Gaussian. C, Start of FF sensitivity. For each FF Gaussian, we took the time at which the temporal envelope of sensitivity reached half of its maximum. $\boldsymbol{D}-\boldsymbol{F}$, Same plots as in $\boldsymbol{A}-\boldsymbol{C}$, but for the suppressive influence as determined by the suppressive Gaussian in the model. Means are indicated by arrows on the $x$-axis. G, Comparison of FF and suppression peak times from model. Units with $R^{2} \geq 0.8$ are solid, open if $<0.2$. There is little correlation ( $r=0.31, p=0.11$ ). $\boldsymbol{H}$, Symbols as in $\mathbf{G}$. Comparison of FF and suppression start times from model. Although start times are better correlated than peak times, the correlation is still weak.

depends on an impending saccade, this saccade-related suppression is often revealed in the FF activity as well.

The components of our model provided an insight into the mechanisms behind the predictive FF activity. We discovered that both the timing of the FF activity and the strength of the FF activity depended on the onset of the saccade. We recognized an increase in sensitivity expressing FF activity, as well as an independent suppressive influence that was also saccade related. Although these two competing influences generally occurred over the same time span, the correlation in their timing was weak, indicating ultimately a similar saccade-related source, but suggesting different mechanisms or pathways.

\section{Discussion}

\section{Temporal characteristics of FF activity}

We concentrated on the temporal characteristics of FF activity in monkey FEF neurons. This activity anticipates the impending saccade by showing sensitivity to stimuli falling in what will be the RF of the neuron after the saccade. This anticipatory activity could contribute to stability of perception across saccades.

We know that FF activity requires both an impending saccade and a stimulus in the FF (Fig. 2). We also confirmed the close relation of FF activity to saccade onset. Together these characteristics establish that FF activity is dependent on the saccade, or perhaps rather on the corollary discharge associated with the saccade (Sommer and Wurtz, 2006).
Two salient findings emerged. First, comparing the time span of the stimulus probes before the saccade to the time span of the FF activity, we found a striking temporal compression of FF activity (Fig. 3). Stimuli flashed over a $70 \mathrm{~ms}$ time span could be compressed into activity spanning only a few milliseconds. Second, many neurons also showed suppression of activity at the time of the saccade, even during FF activity (Fig. 4).

Both FF activity and suppression were intertwined, making it difficult to separate the effects of each. We therefore constructed a simple model that incorporated an $\alpha$ function for the FF probes, a Gaussian increase of sensitivity for the FF, and a Gaussian decrease of activity for suppression. The model determined the time courses of the increase in FF sensitivity and the suppression, and we could infer that FF activity and suppression had about the same average time course (Fig. 7). But considering each neuron (Fig. $7 G, H$ ), it became evident that the time courses of compression and suppression were different, as discussed further later.

\section{Compression of FF activity}

The span of time between the first and the last probe stimulus was frequently substantially longer than the spread of the FF activity-a pronounced temporal compression. FF activity was compressed an average of $64 \%$ relative to the probes. Compression was unmistakable in the majority of neurons that we tested, although the degree of compression varied (Fig. 3D). 
A

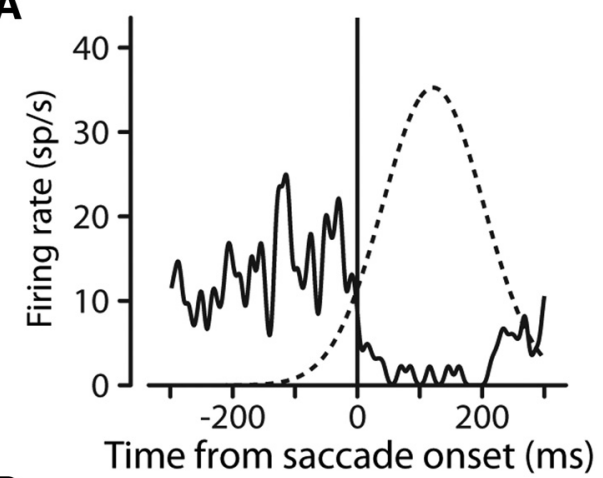

B

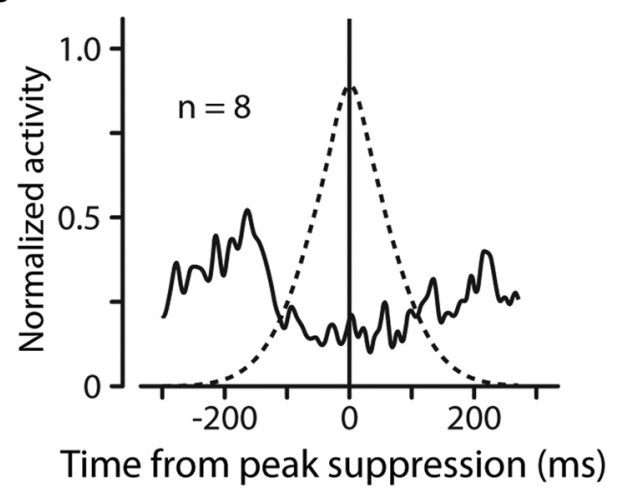

Figure 8. FF sensitivity and suppression follow different time courses. $\boldsymbol{A}$, Suppression depends only on the saccade. For our example neuron (the same neuron in Fig. 2) the solid trace shows the neuron's perisaccadic activity in the absence of any $\mathrm{FF}$ probe. The dashed curve shows the shape of the suppressive component of the model that was fit to the FF activity. The suppression during the saccade-only activity is the same as the suppression that appears during the FF activity. $\boldsymbol{B}$, Average activity (solid line) and suppression (dashed line) for eight neurons using strong fits with substantial suppression. Suppression occurs as predicted by our model.

When we recognized that FF activity is consistently better aligned to saccade onset than FF probe onset, the mechanism of this temporal compression became less of a mystery. If we assume that there is a limited time window of sensitivity for stimuli in the FF and that the window is aligned to the saccade, then only probe activity falling in this window will produce measurable FF activity. Since FF sensitivity for a neuron is fixed in time (relative to the saccade) all activity evoked by probes, regardless of their timing, will appear within this fixed window.

Consider the following analogy. Two neighbors who work together walk to the bus stop, but one leaves home $10 \mathrm{~min}$ after the other. Both, however, get on the same bus, and arrive at work at the same time. Thus the bus "compresses" their commute time by converting the $10 \mathrm{~min}$ between their departures to essentially no difference in their arrivals. But considered from the workers' point of view, the bus actually extends the commute for the early neighbor, in much the same way that an early probe must wait until the FF sensitivity develops for its activity to become visible. Thus compression of departure times can also be viewed as expansion of commute times. This demonstrates that the temporal compression we see in our data is actually a subjective measurement based on the frame of reference: compression of activity timing, or expansion of activity latency. Both result from the alignment, in time, of asynchronous events. But as our time frame is based on the saccade, we will continue to refer to the temporal alignment of probe activity as "compression."

This compression at the neuronal level may be relevant to observations in humans that compression in both space and time occurs for stimuli presented just before saccades (Cicchini et al., 2013). For example, stimuli flashed just before saccades are perceived as falling spatially closer to the saccade target (Matin and Pearce, 1965; Honda, 1989; Dassonville et al., 1995), and interpreted as a compression of space (Ross et al., 1997; Kaiser and Lappe, 2004), Binda et al. (2009) have shown a similar compression in time (or, rather, an expansion of latency); bars flashed just before the saccade were perceived to occur 50-100 ms later. Note that while aspects of the human and monkey experiments differ, both are examining events aligned to the saccade (perceptual or neuronal). In light of this critical parallel between the monkey and human studies, we believe that the compression of FF activity we have shown provides a tantalizing neuronal correlate for temporal compression in human psychophysics (Burr and Morrone, 2012; Cicchini et al., 2013). This comparison suggests that experiments such as ours could be used to explore the neuronal bases of similar psychophysical phenomena.

\section{Suppression of FF activity}

With the aid of our model we identified suppression of activity in most of our 28 neurons, all of which were selected for showing FF activity. We were surprised to find such a prominent suppression, and are unaware of previous reports of suppression in FEF. However, in the plots of FEF FF activity from the review of Sommer and Wurtz (2008), there are notches that might well be suppression. Kusunoki and Goldberg (2003), in their study of the timing of FF activity in LIP, comment that some LIP neurons show saccadic suppression of varying strength, whereas others do not. Again it seems possible that the notch just before the saccade in their plots of averaged LIP activity (their Figures 7 and 8) results from suppression. We may have seen more suppression in our experiments in FEF as elevated FF activity allowed suppression to be seen against a higher firing rate. Thus, it is possible that the suppression we clearly see in frontal eye field has been seen in LIP as well.

FF activity has been shown to depend on the corollary discharge (CD) input to FEF from superior colliculus (Sommer and Wurtz, 2006) and it would seem reasonable that the timing of both FF sensitivity and the concurrent suppression are both determined by the same CD input. However, whereas peak time and duration are similar for the FF and suppression, individual neurons show substantial differences (Fig. 7), implying separate inputs. Both may be produced by a CD, but it might not be the same one, or it might be the same CD but through different pathways. In any case the effects in FEF are of different signs: excitatory for the FF and inhibitory for suppression, so both effects could not arise from identical CD signals in FEF. It should also be noted that FF activity and suppression often begin before the saccade (Fig. 7) so their early influences cannot result from proprioceptive feedback, which begins well after saccade onset. Suppression is also unlikely to result from visual masking, due to our controlled experimental setting (dark room and small, transient stimuli).

Suppression is clear in a number of visual areas (for a review, see Wurtz, 2008), particularly middle temporal (Thiele et al., 2002; Price et al., 2005; Zirnsak et al., 2011), and is considered a candidate neuronal mechanism for blocking the retinal blur during saccades to improve visual stability. Suppression might, however, reduce activity during saccades so that any rebound after the saccade would produce a period of hypersensitivity, making stimuli falling in the RF after the saccade more effective. In this case the suppression during saccades might not produce perceptual suppression during the saccade, but rather produce a period of heightened sensitivity after the saccade. 
Another hypothesis related to perceptual stability across saccades is that stability is maintained at the neuronal level by matching the anticipatory FF activity to the activity in the RF after the saccade (Deubel et al., 2002). A match between FF and RF activities indicates stability; failure indicates changes in the real world. We would expect FF activity to occur first, suppression second, and finally the new RF activity. This, however, is not what happens. Suppression occurs independently of FF activity: before, during, or after. This argues against the function of such suppression being necessary for presaccadic and postsaccadic comparisons.

Our study shows that suppression and FF sensitivity are both temporally related to saccades. We submit that temporal compression is simply an alignment of asynchronous presaccadic events to a saccade-related envelope of FF sensitivity. We also show that the time course of suppression is independent of FF sensitivity. This indicates that FF sensitivity (which underlies temporal compression) is not necessarily precipitated by the same signal that triggers suppression during the saccade. Although corollary discharge in preparation of saccades may play a role in both suppression and compression, it is evident that it is not the same CD signal, or at least not the same pathway.

\section{References}

Binda P, Cicchini GM, Burr DC, Morrone MC (2009) Spatiotemporal distortions of visual perception at the time of saccades. J Neurosci 29:1314713157. CrossRef Medline

Bruce CJ, Goldberg ME, Bushnell MC, Stanton GB (1985) Primate frontal eye fields. II. Physiological and anatomical correlates of electrically evoked eye movements. J Neurophysiol 54:714-734. Medline

Burr DC, Morrone MC (2012) Constructing stable spatial maps of the world. Perception 41:1355-1372. CrossRef Medline

Cavanaugh JR, Bair W, Movshon JA (2002) Nature and interaction of signals from the receptive field center and surround in macaque $\mathrm{V} 1$ neurons. J Neurophysiol 88:2530-2546. CrossRef Medline

Cicchini GM, Binda P, Burr DC, Morrone MC (2013) Transient spatiotopic integration across saccadic eye movements mediates visual stability. J Neurophysiol 109:1117-1125. CrossRef Medline

Dassonville P, Schlag J, Schlag-Rey M (1995) The use of egocentric and exocentric location cues in saccadic programming. Vision Res 35:21912199. CrossRef Medline

Deubel H, Schneider WX, Bridgeman B (2002) Transsaccadic memory of position and form. Prog Brain Res 140:165-180. CrossRef Medline

Duhamel JR, Colby CL, Goldberg ME (1992) The updating of the representation of visual space in parietal cortex by intended eye movements. Science 255:90-92. CrossRef Medline

Hays AVR, Richmond BJ, Optican LM (1982) A UNIX-based multiple process system for real-time data acquisition and control. WESCON Conf Proc 2:1-10.

Hoel P, Port S, Stone C (1971) Introduction to statistical theory. Boston: Houghton-Mifflin.

Honda H (1989) Perceptual localization of visual stimuli flashed during saccades. Percept Psychophys 45:162-174. CrossRef Medline

Kaiser M, Lappe M (2004) Perisaccadic mislocalization orthogonal to saccade direction. Neuron 41:293-300. CrossRef Medline

Kusunoki M, Goldberg ME (2003) The time course of perisaccadic receptive field shifts in the lateral intraparietal area of the monkey. J Neurophysiol 89:1519-1527. Medline

Matin L, Pearce DG (1965) Visual perception of direction for stimuli flashed during voluntary eye movements. Science 148:1485-1488. CrossRef Medline

Price NS, Ibbotson MR, Ono S, Mustari MJ (2005) Rapid processing of retinal slip during saccades in macaque area MT. J Neurophysiol 94:235246. CrossRef Medline

Ross J, Morrone MC, Burr DC (1997) Compression of visual space before saccades. Nature 386:598-601. CrossRef Medline

Sommer MA, Wurtz RH (2000) Composition and topographic organization of signals sent from the frontal eye field to the superior colliculus. J Neurophysiol 83:1979-2001. Medline

Sommer MA, Wurtz RH (2006) Influence of the thalamus on spatial visual processing in frontal cortex. Nature 444:374-377. CrossRef Medline

Sommer MA, Wurtz RH (2008) Brain circuits for the internal monitoring of movements. Annu Rev Neurosci 31:317-338. CrossRef Medline

Thiele A, Henning P, Kubischik M, Hoffmann KP (2002) Neural mechanisms of saccadic suppression. Science 295:2460-2462. CrossRef Medline

Umeno MM, Goldberg ME (1997) Spatial processing in the monkey frontal eye field. I. Predictive visual responses. J Neurophysiol 78:1373-1383. Medline

Wurtz RH (2008) Neuronal mechanisms of visual stability. Vision Res 48 : 2070-2089. CrossRef Medline

Zirnsak M, Gerhards RG, Kiani R, Lappe M, Hamker FH (2011) Anticipatory saccade target processing and the presaccadic transfer of visual features. J Neurosci 31:17887-17891. CrossRef Medline 